\title{
Relation between quantum statistics of phonons and scattered light
}

\author{
Alexander S. Shumovsky ${ }^{\text {a,b }}$ and B. Tanatar ${ }^{\text {a }}$ \\ - Department of Physics, Bilkent University, Bilkent, 06533 Ankara, Turkey \\ b Bogolubov Laboratory of Theoretical Physics, Joint Institute for Nuclear Research, \\ P.O. Box 79, Moscow 101000, Russian Federation
}

Received 29 July 1993; revised manuscript received 27 September 1993; accepted for publication 28 September 1993 Communicated by B. Fricke

\begin{abstract}
The resonance model of the Raman process with the generation of a Stokes component is solved exactly. The asymptotic behavior of the solution is discussed. Mandel's $Q$-factor is calculated as a function of time both for the Stokes and the Rayleigh components for their dependence on the quantum statistical properties of the vibration mode (phonons). A qualitative difference between the cases of uncorrelated and correlated phonons is found which may have interesting experimental implications.
\end{abstract}

\section{Introduction}

During the last years quantum statistical properties of scattered light in the Raman process have attracted considerable interest [1,2]. In particular, the anticorrelation between the Stokes and Rayleigh lines in the resonance scattering have been examined $[3,4]$, and the generation of squeezed light has been considered [5-7]. At the same time, strong quantum fluctuations of energy have been observed experimentally $[8,9]$.

It is known that Raman scattering is an example of an optical parametric process in which one of the interacting waves is a medium vibration mode of boson type (phonons) [10]. In the case of condensed matter such a mode is usually in thermal equilibrium with a given temperature. The state of that mode is determined by different mechanisms of microscopic interactions in the medium and in some cases can lead to a strong number fluctuation [11]. An example is provided by a polariton-type system in which the equilibrium state is a squeezed one [12]. Undoubtedly, the statistical properties of the vibration mode must have influence on the statistics of the scattered light.

In the present paper we consider the quantum properties of scattered light and its dependence on the type of statistical distribution function of the vi- bration mode in Raman scattering. For simplicity, we suppose the resonance steady state process with the generation of an inelastic Stokes component only. The initial state of the Rayleigh mode is assumed to be a number state, while the vibration mode can be initially in a number state or in a squeezed vacuum state. The Stokes field is initially in the vacuum state. The simplest model of three bounded oscillators is used for the description of the process under consideration [13]. Using the representation of the Schrödinger equation in terms of new orthogonal polynomials $[14,15]$, we examine the dynamics of Mandel's factor of scattered light, and show the qualitative difference between the two choices of the initial states of the vibration mode.

The rest of this paper is organized as follows. We first introduce the model Hamiltonian for which we calculate the dynamical properties. The evaluation of the eigenvalues and eigenfunctions, and a discussion of how to construct the time-dependent Mandel factor is given in the next section. We then present our results and conclude with an emphasis on the experimental implications.

\section{Model and its eigenvalue spectrum}

The aim of this study is the calculation of the time- 
dependent Mandel factor defined by the following expression,

$Q_{t}(g) \equiv \frac{V_{t}\left(c_{g}^{+} c_{g}\right)-\left\langle c_{g}^{+} c_{g}\right\rangle_{t}}{\left\langle c_{g}^{+} c_{g}^{-}\right\rangle_{t}^{-}}$

Here $c_{g}^{+}$and $c_{g}$ are the Bose operators, $V_{t}\left(c_{g}^{+} c_{g}\right)$ is the time-dependent number variance, and \langle\rangle$_{t}$ denotes a time-dependent expectation value. It is positive in the case of super-Poisson statistics and negative for a sub-Poisson number distribution. The zero value corresponds to the coherent state (Poisson distribution).

The time dependence of the functions on the right hand side of eq. (1) is given by the dynamics of our model Hamiltonian,

$H=\sum_{g} \omega_{g} c_{g}^{+} c_{g}+\gamma\left(c_{\mathrm{v}}^{+} c_{\mathrm{s}}^{+} c_{\mathrm{r}}+\right.$ h.c. $)$,

where the index $g=\mathrm{v}, \mathrm{s}$ or $\mathrm{r}$ denotes the vibration, Stokes, or Rayleigh modes, respectively. We will assume the resonance condition

$\omega_{\mathrm{r}}=\omega_{\mathrm{s}}+\omega_{\mathrm{v}}$

in the subsequent considerations. Because of the conservation law [13] $[N, H]=0$, where

$N=c_{\mathrm{r}}^{+} c_{\mathrm{r}}+\frac{1}{2}\left(c_{\mathrm{s}}^{+} c_{\mathrm{s}}+c_{\mathrm{v}}^{+} c_{\mathrm{v}}\right)$,

an exact eigenstate of the Hamiltonian of eq. (2) can be chosen as

$|n, m\rangle=\sum_{j=0}^{n} \lambda_{j}^{n, m}|n-j\rangle_{\mathrm{r}}|j\rangle_{\mathrm{s}}|m+j\rangle_{\mathrm{v}}$,

where |\rangle$_{g}$ is the number state of the gth mode. The coefficients $\lambda_{j}^{n, m}$ in eq. (4) are determined by the recursion relation [15]

$$
\begin{aligned}
& \lambda_{j+1}^{n, m}[(n-j)(j+1)(m+j+1)]^{1 / 2} \\
& \quad=x^{n, m} \lambda_{j}^{n, m}-\lambda_{j-1}^{n, m}[(n-j+1) j(m+j)]^{1 / 2},
\end{aligned}
$$

together with the normalization condition

$\sum_{j=0}^{n}\left|\lambda_{j}^{n, m}\right|^{2}=1$.

Here $x^{n, m}=\left(E^{n, m}-\omega_{\mathrm{r}} n-\omega_{\mathrm{v}} m\right) / \gamma$, and $E^{n, m}$ is an eigenvalue of Hamiltonian (2) corresponding to the eigenstate given in eq. (4). The above relation (5) can also be represented by the equivalent expression

$P_{j+1}^{n, m}(x)=x P_{j}^{n, m}(x)-q_{j}^{n, m} P_{j-1}^{n, m}(x)$, defining some orthogonal polynomials $P_{j}^{n, m}(x)$. Here

$q_{j}^{n, m}=(n-j+1) j(m+j)$.

These polynomials may also be defined through the differential equation

$$
\begin{aligned}
& t^{3} \frac{\partial^{2} F}{\partial t^{2}}-\left[1+t^{2}(n-m-2)\right] \frac{\partial F}{\partial t} \\
& \quad+[x-\operatorname{tn}(m+1)] F=0
\end{aligned}
$$

for the following generating function,

$F(x, t)=\sum_{j=0}^{\infty} P_{j}^{n, m}(x) \frac{t^{j}}{j !}$.

Let us represent the polynomials $P_{j}^{n, m}(x)$ in the form $P_{j}^{n, m}(x)=\sum_{l=0}^{j} \xi_{l}^{n, m}(j) x^{l}$.

Then for the coefficients $\xi$ in the above equation we get

$$
\begin{aligned}
& \xi_{j-2 s+1}^{n, m}(j)=0, \\
& \xi_{j-2 s}^{n, m}(j)=-\sum_{k=2 s-1}^{j-1} q_{k}^{n, m} \xi_{k-2 s+1}^{n, m}(k-1) .
\end{aligned}
$$

Because of this relation it is not difficult to express these coefficients $\xi$ in terms of the Bernoulli polynomials [15].

Using the polynomials $P_{j}^{n, m}(x)$ we can represent the equation for the eigenvalues in the form

$P_{n+1}^{n, m}(x)=0$.

Taking into account the relations given in eq. (7), one can observe that (i) for any fixed $n$ and $m$ eq. (8) has exactly $n+1$ real solutions; (ii) the set of solutions $\left\{x_{l}^{n, m}\right\}$ is ordered symmetrically with respect to the zero value $x^{n, m}=0$; (iii) the value $x^{n, m}=0$ for which $E_{n, m}=\omega_{\mathrm{r}} n+\omega_{\mathrm{v}} m$ is the root of eq. (8) for even $n$ only; and (iv) between any two neighboring roots $x_{l}^{n, m}$ and $x_{l+1}^{n, m}$ is situated only one root of eq. (8). We note that the above expression (8) determines the eigenvalues of some Jacobi matrix. For a special case the problem of diagonalization of the corresponding matrix was solved numerically by Walls and Barakat [16].

With the aid of eqs. (5) and (6) the coefficients 
of the eigenstate given by eq. (4) are represented in the form

$$
\begin{aligned}
& \lambda_{j}^{n, m}\left(x_{l}\right)=\lambda_{0}^{n, m} P_{j}^{n, m}\left(x_{l}\right) \\
& \quad \times\left[(j !)^{3}\left(\begin{array}{c}
n \\
j
\end{array}\right)\left(\begin{array}{c}
m+j \\
j
\end{array}\right)\right]^{-1 / 2},
\end{aligned}
$$

where $x_{l} \equiv x_{l}^{n, m}$ is any solution of eq. (8). The coefficient $\lambda_{0}^{n, m}$ is determined by the normalization condition. For example, the eigenstate corresponding to the solution $x^{n, m}=0$ for even $n$ is described by the following set of coefficients,

$\lambda^{n, m}=\lambda_{0}^{n, m}\left\{1,0, \frac{\xi_{0}^{n, m}(2)}{F_{2}^{n, m}}, 0, \frac{\xi_{0}^{n, m}(4)}{F_{4}^{n, m}}, \ldots, 0, \frac{\xi_{0}^{n, m}(n)}{F_{n}^{n, m}}\right\}$

where

$\xi_{0}^{n, m}(2 p)=(2 p-1) ! !(2 p) ! ! \frac{(m+2 p-1) ! !}{(m-1) ! !}$

and

$F_{2 p}^{n, m}=\left[[(2 p) !]^{3}\left(\begin{array}{c}n \\ 2 p\end{array}\right)\left(\begin{array}{c}m+2 p \\ 2 p\end{array}\right)\right]^{-1 / 2}$.

We stress that the same polynomials and polynomial equations arise in the more general case of nonlinear interaction of any order $p \geqslant 2$ of the type

$H_{\mathrm{int}}=\gamma\left(a_{0}^{+} \prod_{k=1}^{p} a_{k}+\prod_{k=1}^{p} a_{k}^{+}\right)$

(decay of one boson into $p$ bosons). The only change here is the parameter $q_{f}^{(n)}$ in eq. (6). For instance, in the case when all $k$ th modes are initially in the vacuum state, one gets $q_{j}^{(n)}=j^{p}(n-j+1)$.

\section{Asymptotics of eigenvalues}

The representation of the eigenvalue problem in terms of the polynomials $P(x)$ allows us to estimate the asymptotic behavior of the eigenvalues $E^{n, m}$ for large $n$ and $m$. Apart from providing us with useful information on the numerical calculations, the investigation of the asymptotic behavior leads also to some interesting properties of the model which we discuss below.

From the results of the previous section it follows that

$$
\begin{aligned}
n & {\left[x_{\max }^{n, m}\right]^{2} \geqslant \sum_{j=0}^{n}\left[x_{j}^{n, m}\right]^{2} \geqslant \sum_{j=0}^{n} q_{j}^{n, m} } \\
& =\frac{1}{12} n(n+1)\left[n^{2}+n(3+2 m)+4 m+2\right],
\end{aligned}
$$

where $x_{\max }$ is the maximum root of eq. (8) at given $n$ and $m$. Because of the above established symmetry of the solutions of eq. (8) we obtain

$$
\begin{aligned}
E_{\min }^{n, m} & \leqslant \omega_{\mathrm{r}} n+\omega_{\mathrm{v}} m \\
& -\frac{\gamma}{2 \sqrt{3}} \sqrt{(n+1)\left[n^{2}+n(2 m+3)+4 m+2\right]},
\end{aligned}
$$

which leads to the asymptotic behavior

$$
E_{\min }^{n, m} \leqslant \omega_{\mathrm{r}} n+\omega_{\mathrm{v}} m-\frac{\gamma}{2 \sqrt{3}} \sqrt{n^{3}+2 n^{2} m}
$$

for large $n$ and $m$. A similar estimation of the lowest eigenvalue for the special case $m=0$ was given previously $[11,14]$. It follows from the inequality of eq. (10) that starting from some numbers $n_{0}$ and $m_{0}$, we have at least one branch of collective excitations with negative energy, while the energy of the vacuum state $E^{0,0}=0$. Moreover, $E_{\min }^{n, m} \rightarrow-\infty$ when $n \rightarrow \infty$. Thus, the spectrum of eigenvalues of the problem under consideration becomes unstable with increasing $n$.

The inequality of eq. (10) gives us an estimate as an upper bound to $E_{\min }^{n, m}$. Using the Frobenius theorem [17] one can also construct a lower bound,

$x_{\min }^{n, m} \leqslant \max _{k}\left(\sqrt{q_{k-1}^{n, m}}+\sqrt{q_{k}^{n, m}}\right) \leqslant \frac{4}{3 \sqrt{3}} \sqrt{n^{3}+2 n^{2} m}$,

which leads to

$$
\begin{gathered}
\omega_{\mathrm{r}}+\omega_{\mathrm{v}} m-\frac{4 \gamma}{3 \sqrt{3}} \sqrt{n^{3}+2 n^{2} m} \leqslant E_{\min }^{n, m} \\
\leqslant \omega_{\mathrm{r}} n+\omega_{\mathrm{v}} m-\frac{\gamma}{2 \sqrt{3}} \sqrt{n^{3}+2 n^{2} m}
\end{gathered}
$$

for large $n$ and $m$. Because of these estimates from above and below, we have the exact asymptotic behavior for the minimum eigenvalue at fixed $n$ and $m$. Due to the symmetry of the roots of eq. (8) similar asymptotics can also be derived for the maximum eigenvalue. We summarize the asymptotic behavior of the minimum and maximum eigenvalues below. 


$$
\begin{aligned}
& E_{\min }^{n, m} \sim-\gamma n^{3 / 2}, \quad n \rightarrow \infty, m \text { fixed, } \\
& E_{\max }^{n, m} \sim \gamma n^{3 / 2}, \quad n \rightarrow \infty, m \text { fixed }, \\
& E_{\min }^{n, m} \sim \omega_{\mathrm{v}} m-\gamma \mathrm{O}\left(m^{1 / 2}\right), \quad m \rightarrow \infty, n \text { fixed, } \\
& E_{\max }^{n, m} \sim \omega_{\mathrm{v}} m+\gamma \mathrm{O}\left(m^{1 / 2}\right), \quad m \rightarrow \infty, n \text { fixed } .
\end{aligned}
$$

From the above asymptotic behavior one readily observes that only $n$ is a critical parameter for stability of the spectrum of elementary excitations in the present model. In this connection, we remark that increasing $n$ means an increase in the number of photons in the Rayleigh mode. But at high intensity of the Rayleigh mode at $t=0$, it is necessary to take into account the generation of anti-Stokes phonons. It is then reasonable to suppose that the inclusion of the anti-Stokes process into the model Hamiltonian would stabilize the spectrum of collective excitations.

\section{Time dependent Mandel factor}

Using the results of previous sections we can now express the exact time dependent wave function of Hamiltonian (2) in the form

$$
\begin{aligned}
& \psi(t)=\sum_{n, m=0}^{\infty} \exp \left[-\mathrm{i}\left(n \omega_{\mathrm{r}}+m \omega_{\mathrm{v}}\right) t\right] \\
& \times \sum_{l=1}^{n+1} C_{l}^{n, m} \exp \left(-\mathrm{i} \gamma x_{l}^{n, m} t\right) \\
& \times \sum_{j=0}^{n} \lambda_{j}^{n, m}\left(x_{l}^{n, m}\right)|n-j\rangle_{\mathrm{r}}|j\rangle_{\mathrm{s}}|m+j\rangle_{\mathrm{v}} .
\end{aligned}
$$

Here we have enumerated the roots $x_{l}^{n, m}$ of eq. (8) starting from the maximum value. The coefficients $C_{l}^{n, m}$ are determined by the initial conditions so that

$$
\begin{aligned}
\sum_{l=1}^{n+1} C_{l}^{n, m} \lambda_{j}^{n, m}\left(x_{l}\right) & =p_{n} f_{m}, & & \text { if } j=0, \\
& =0, & & \text { otherwise . }
\end{aligned}
$$

According to the setting of the problem given in section 1 we must put here

$p_{n}=\delta_{n n}$

for the initial state of the Rayleigh mode, and

$$
\begin{aligned}
f_{m} & =\delta_{m m^{\prime}}, \\
& =\frac{1}{\sqrt{n ! \mu}}(\nu / 2 \mu)^{n / 2} H_{m}(0)
\end{aligned}
$$

for the number and squeezed vacuum initial states of the vibration mode, respectively. Here $\mu$ and $\nu$ are the squeezing parameters and $H(x)$ is the Hermite polynomial [18].

Now all time dependent terms in the Mandel factor given in eq. (1) must be calculated as the corresponding expectation values with the time dependent wave function (eqs. (13), (14)).

The results of our calculations are presented in figs. 1 and 2 for Mandel' $Q$-factors of the Rayleigh and Stokes modes, respectively. In fig. 1a we show the Rayleigh mode Mandel factor $Q_{\mathrm{r}}(t)$ for the initial number state of the vibration mode $(m=2)$, and different values of the initial number of photons $n=2$ (solid line) and $n=20$ (dotted line) in the Rayleigh mode. One can see that the increase of $n$ in this case leads to a periodical change of statistics of elastic scattered photons from the sub-Poissonian to the super-Poissonian statistics.

A qualitatively different behavior is observed when the vibration mode is initially in a squeezed vacuum state (fig. 1b). In that case as $n$ increases for a fixed mean number of quanta in the vibration mode $|\nu|^{2}$, the statistics of scattered light becomes completely super-Poissonian without any change of sign of Mandel's $Q$-factor. Figure 2 shows Mandel's $Q$-factor for the Stokes mode. Qualitatively the same behavior is observed also for the Stokes mode as discussed before.

\section{Conclusion}

We have obtained a qualitative difference in the quantum statistical properties of scattered light depending on the statistics of the vibration mode. Our choice of the initial state of the vibration mode can be considered as simulating the harmonic (uncorrelated) and strongly correlated vibrations in a medium. Hence, the experimental investigation of the quantum statistical properties of scattered light in the Raman correlation spectroscopy with a number state (or a strong sub-Poissonian) incident light may 

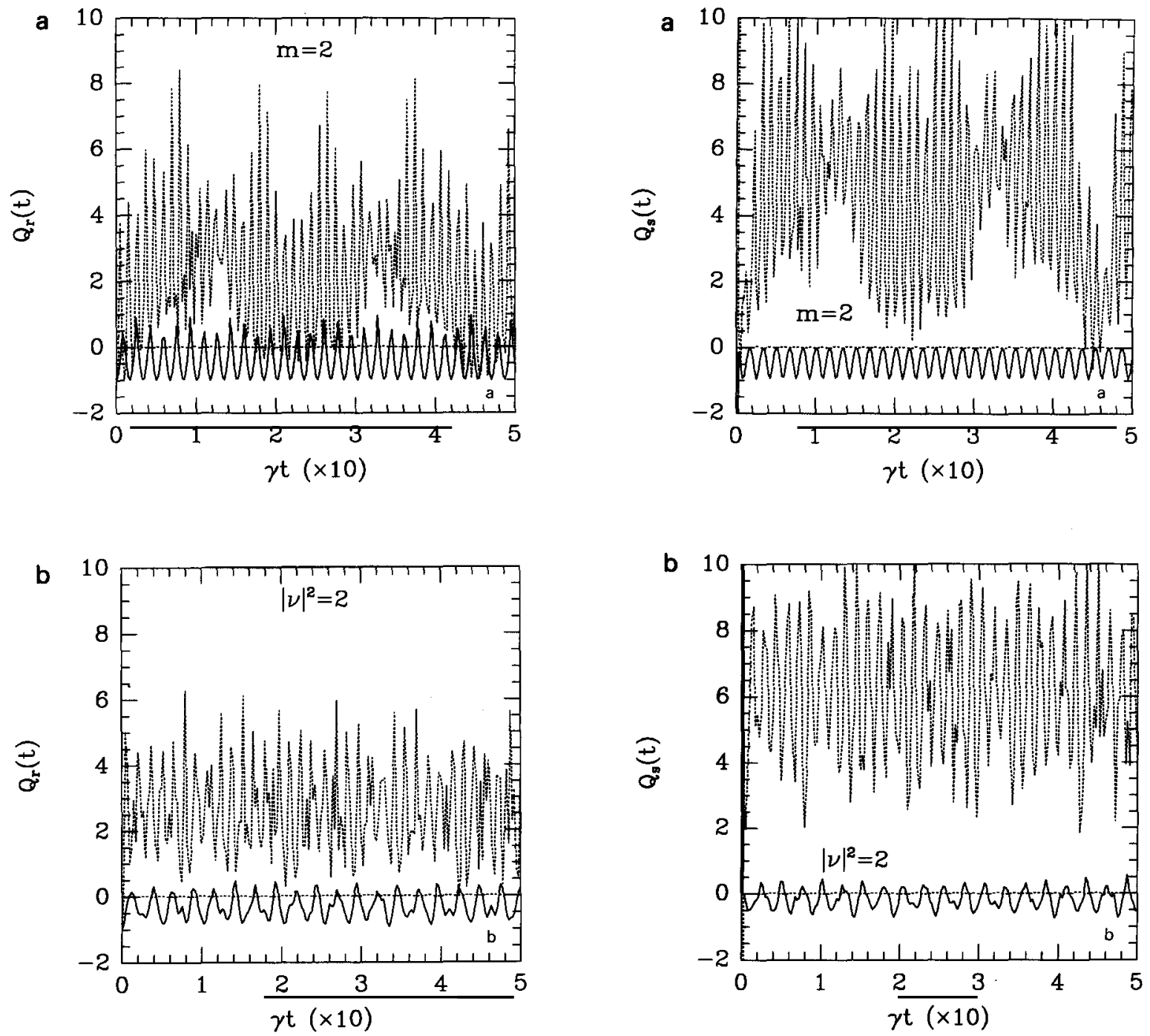

Fig. 1. (a) Time dependent Mandel factor $Q_{r}(t)$ for the Rayleigh mode when the vibration mode is initially in the number state ( $m=2$ ), and the initial number of photons $n=2$ (solid line) and $n=20$ (dotted line) for the Rayleigh mode. (b) Time dependent Mandel factor $Q_{\mathrm{r}}(t)$ for the Rayleigh mode when the vibration mode is initially in the squeezed state $|\nu|^{2}=2.0$, and the initial number of photons $n=2$ (solid line) and $n=20$ (dotted line) for the Rayleigh mode.

yield important information on the correlations in the medium as well as in the molecules.

It is not difficult to see from figs. 1 and 2 that the collapse-revival phenomenon occurs in the system for sufficiently large $n$. Since an increase in $n$ implies an increase in the number of terms in the sum of eq.

Fig. 2. (a) Time dependent Mandel factor $Q_{\mathrm{s}}(t)$ for the Stokes mode when the vibration mode is initially in the number state ( $m=2$ ), and the initial number of photons $n=2$ (solid line) and $n=20$ (dotted line) for the Rayleigh mode. (b) Time dependent Mandel factor $Q_{s}(t)$ for the Stokes mode when the vibration mode is initially in the squeezed state $|\nu|^{2}=2.0$, and the initial number of photons $n=2$ (solid line) and $n=20$ (dotted line) for the Rayleigh mode.

(10), it is not surprising to observe the collapserivival patterns as in the case of the JaynesCummings model $[19,20]$. It should be noted that a similar behavior was obtained in the numerical calculations of Drobny and Jex [21], for the case of the initial coherent state of the Rayleigh mode. In this 
connection we emphasize that the collapse-revival phenomenon is a general property of the model described by Hamiltonian (2) irrespective of the initial state of the Rayleigh mode. Some other cases are also considered in ref. [15].

\section{Acknowledgement}

A.S. would like to thank Professors C. Bowden, R. Bullough, S. Carusotto, O. Keller, F. Persico and V. Rupasov for fruitful discussions.

\section{References}

[1] A.S. Shumovsky and Tran Quang, in: Interaction of electromagnetic field with condensed matter, eds. N.N. Bogolubov, A.S. Shumovsky and V.I. Yukalov (World Scientific, Singapore, 1990) p. 103.

[2] J. Mostowski and M.G. Raymer, in: Contemporary nonlinear optics, eds. G.P. Agarval and R.W. Boyd (Academic Press, New York, 1992) p. 187.

[3] G. Agarwal and S. Jha, Z. Phys. B 25 (1979) 391.
[4] N. Bogolubov Jr., A. Shumovsky and Tran Quang, J. Phys. (Paris) 48 (1987) 1671.

[5] H.P. Yuen, Phys. Rev. A 13 (1976) 2226.

[6] A. Shumovsky and Tran Quang, Phys. Lett. A 131 (1988) 471.

[7] C.C. Gerry and J.H. Eberly, Phys. Rev. A 42 (1990) 6805.

[8] I.A. Walmsley and M.G. Raymer, Phys. Rev. A 33 (1986) 382.

[9] M.D. Duncan, R. Mahon, L.L. Tankersley and J. Reintjes, J. Opt. Soc. Am. B 7 (1990) 1336.

[10] Y.R. Shen, The principles of nonlinear optics (Wiley, New York, 1984).

[11] A.S. Shumovsky, in: Modern nonlinear optics, ed. M. Evans (Wiley, New York, 1993).

[12] A. Chizhov, B. Govorkov Jr. and A. Shumovsky, Int. J. Mod. Phys. B, to be published.

[13] S. Carusotto, Phys. Rev. A 40 (1989) 1848.

[14] Yu. Orlov, I. Pavlotsky, A. Shumovsky, V. Suslin and V. Vedenyapin, Int. J. Mod. Phys. B, to be published.

[15] A. Shumovsky and B. Tanatar, Phys. Rev. A, to be published.

[16] D.F. Walls and R. Barakat, Phys. Rev. A 1 (1970) 446.

[17] M. Marcus and H. Minc, A survey of matrix theory and matrix inequalities (Allyn and Bacon, Boston, 1964).

[18] R. Loudon and P.L. Knight, J. Mod. Opt. 34 (1987) 709.

[19] H.I. Yoo and J.H. Eberly, Phys. Rep. 118 (1985) 239.

[20] Fam Le Kien and A.S. Shumovsky, Int. J. Mod. Phys. B 5 (1991) 2287.

[21] G. Drobny and I. Jex, Phys. Rev. A 46 (1992) 499. 